\title{
Influence Du Poids Maternel Sur L'evolution De La Grossesse Chez Les Gestantes Dans La Ville De Parakou En 2015 Au Benin
}

\author{
Achille Awadé Afoukou Obossou
}

UER en gynécologie Obstétrique, Faculté de Médecine, Université de Parakou (Bénin ) Angéline Josiane Tonato Bagnan

UER en Gynécologie Obstétrique, Faculté des sciences de la Santé,

Université d'Abomey Calavi (Bénin)

Fanny Maryline Nouessèwa Hounkponou Ahouingnan

UER en gynécologie Obstétrique, Faculté de Médecine, Université de Parakou (Bénin )

Benjamin Ignace Bodounrin Hounkpatin

UER en Gynécologie Obstétrique, Faculté des sciences de la Santé, Université d'Abomey Calavi (Bénin)

Rachidi Imorou Sidi Kabibou Salifou

\section{Mahublo Vinadou Vodouhe}

Edna Mirabelle Oza

UER en gynécologie Obstétrique, Faculté de Médecine,

Université de Parakou (Bénin )

\section{François Djrolo}

Uer En Médecine Et Spécialités Médicales, Faculté De Médecine, Université De Parakou (Bénin )

doi: 10.19044/esj.2016.v12n27p218 URL:http://dx.doi.org/10.19044/esj.2016.v12n27p218

\section{Résumé}

Introduction:The World Health Organization considers for many years obesity as a pandemic which affects as well the developed countries as the developing ones.The consequences of the overweight on the medical, gynaeco-obstetrical, and surgical plan are no longer demonstrating.

Objective:The current paper aims to study the influence of the weight of the mother on the evolution of the pregnancy with the pregnant women in the town of Parakou.

Material and methodologicalapproaches: It was about a descriptive transversal study for an analytical purpose with forecast data collection. It 
has been conducted on a period of six (06) months going from 23rd of February to 31st of August 2015 and had covered 272 pregnant women in the town of Parakou.The sampling probabilistic method has been used according to the survey technique.

Results:The average age of the surveyed women was about 26, $1 \pm 5,8$ years old with the extremes of 14 and 51 years old. The predominance of the overweight and the obesity with the pregnant women in Parakou was respectively about $34,9 \%$ and $15,8 \%$. The diabetes pregnant woman, the exceeding of term and the macrosomy were statistically associated with obesity. The diabetes pregnant women, the exceeding of term, the resuscitation at birth were statistically associated with overweight.

Conclusion: The fight against overweight is one of the great challenges of this century. It has some consequences on obstetrical plan. A planning of pregnancies with the patients of overweight would then permit a clear improvement of the undertaking of their social securitywithin the maternities and an improvement of the maternal, perinatal and neonatal indicators.

Keywords: Influence, overweight, pregnancy, Parakou, Republic of Bénin

\section{Abstract}

Introduction : L'OMS considère depuis plusieurs années l'obésité comme une pandémie qui touche aussi bien les pays industrialisés que les pays du sud. Les conséquences de l'excès pondéral sur le plan médical, gynéco - obstétrical et chirurgical ne sont plus à démontrer.

Objectif : Etudier l'influence du poids maternel sur l'évolution de la grossesse chez les gestantes dans la ville de Parakou.

Matériel et méthodes : il s'agissait d'une étude transversale descriptive à visée analytique avec collecte prospective des données Elle s'est déroulée sur une période de 6 mois allant du 23 février au 31 août 2015 et avait inclu 272 gestantes de la ville de Parakou. La méthode d'échantillonnage probabiliste selon la technique de sondage à deux degrés a été utilisée.

Résultats : L'âge moyen des femmes enquêtées était de 26,1 1 5,8 ans avec des extrêmes de 14 et 51 ans. La prévalence de surpoids et de l'obésité chez les gestantes à Parakou en 2015 étaient respectivement de 34,9\% et de $15,8 \%$. Le diabète gestationnel, le dépassement de terme, la macrosomie étaient statistiquement associés à l'obésité. Le diabète gestationnel, le dépassement de terme, la réanimation à la naissance étaient statistiquement associés au surpoids.

Conclusion : La lutte contre l'excès de poids est un des grands défis de ce siècle. Il engendre des conséquences sur le plan obstétrical. Une programmation des grossesses chez les patientes en excès de poids 
permettrait alors une amélioration nette de leur prise en charge et une amélioration des indicateurs maternels, périnataux et néonataux.

Mots - clés : Influence, excès pondéral, grossesse, Parakou, Bénin

\section{Introduction}

Depuis plusieurs années, l'Organisation Mondiale de la Santé (OMS) considère l'obésité comme une pandémie qui touche aussi bien les pays industrialisés que les pays en voie de déloppement (Cohen, 2010). La prévalence de l'obésité dans le monde a doublé depuis 1980. En 2008, 1,5 milliards d'adultes étaient en excès de poids dont 200 millions d'hommes et 300 millions de femmes. En 2014, l'excès pondéral touchait plus de 1,9 milliard d'adultes dont plus de 600 millions étaient obèse. D'après les prédictions, plus de la moitié de la population adulte deviendra obèse ou en surpoids d'ici 2030 dans le Monde (Ducarme et al., 2007). L'excès pondéral est un important facteur de risque de maladies chroniques tels que les maladies cardiovasculaires, le diabète, les troubles musculo-squelettiques, certains cancers de l'endomètre, du sein et du côlon (Grossetti et al. , 2004). Sur le plan obstétrical, La grossesse des femmes en excès pondéral est plus fréquemment marquée par des complications maternelles et néonatales (Grossetti et al., 2004 ; Eicholzer, 2006).

En Afrique subsaharienne les données sur les répercussions obstétricales de l'obésité sont rares. Au Bénin, une étude faite dans une population de gestantes à l'hôpital universitaire de référence de Cotonou en 2002 avait retrouvé le diabète sucré, l'accouchement par césarienne, la macrosomie associés à l'obésité (Djrolo et al., 2002). L'objectif du présent travail était donc de déterminer la prévalence de l'obésité et du surpoids dans une population de gestantes de la région septentrionale du Bénin et d'étudier l'influence de l'obésité sur l'évolution de la grossesse.

\section{Materiel et methodes}

L'étude s'est déroulée sur une période de 6 mois allant du 23 février au 31 août 2015 dans la ville de Parakou, partie septentrionale du Bénin. Il s'agissait d'une étude transversale descriptive à visée analytique avec collecte prospective des données. La population d'étude était constituée de l'ensemble des gestantes dont le terme gestationnel révolu était supérieur ou égal à 24 semaines d'aménorrhée(SA) et inférieur ou égal à 28 semaines d'aménorrhée dont le poids et la taille avaient été documentés au premier trimestre de la grossesse. Ont été exclues, les femmes ayant refusé de participer à l'enquête ; celles dont le poids n'avait pas été pris au premier trimestre ; ou celles ayant été perdues de vue. La méthode d'échantillonnage probabiliste selon la technique de sondage à deux degrés a été utilisée. Le 
premier degré a consisté à sélectionner à l'aide d'un tirage aléatoire simple sans remise $50 \%$ des formations sanitaires offrant les services de Consultation Prénatales (CPN) dans la commune de Parakou. Le tirage a été fait avec le logiciel statistique ENA. Le deuxième degré a consisté à un recrutement systématique de toutes les gestantes consultant dans les formations sanitaires retenues et qui répondaient à nos critères d'inclusion pendant la période de collecte. Au total, l'étude a inclus 272 gestantes. L'indice de masse corporelle a été déterminé par le rapport du poids (en $\mathrm{Kg}$ ) et de la taille (en mètre au carré). Il est considéré comme normal lorsqu'il est compris entre 20 et 24,9. Entre 25 et 29,9, la femme est considérée comme en surcharge pondérale et à partir de 30 comme obèse (WHO, 1997). La protéinurie a été déterminée de façon semi-quantitative par les bandelettes d'albustix et elle a été considérée comme significative pour définir une pré éclampsie à partir de deux croix. Les femmes étaient considérées comme hypertendues lorsque la tension artérielle dépassait $140 \mathrm{~mm} \mathrm{Hg}$ pour la systolique et $90 \mathrm{~mm} \mathrm{Hg}$ pour la diastolique. Nous avons posé le diagnostic de diabète gestationnel à partir de la glycémie à jeûn $\geq 0,92 \mathrm{~g} / 1 \mathrm{chez}$ la femme enceinte dont le terme se situe entre 24 SA et 28 SA (Puech, 2010). Les accouchements ont été considérés à terme entre $37 \mathrm{SA}$ et $42 \mathrm{SA}$. La grossesse était donc en dépassement de terme au-delà de 42SA. La macrosomie fœtale était définie à partir de $4000 \mathrm{~g}$ et l'hypotrophie fœtale, à partir d'un poids inférieur à $2500 \mathrm{~g}$. Les données ont été saisies avec EPI data 3.1 et analysées avec le logiciel EPI Info 7. Les fréquences ont été comparées avec le test exact de Fischer ou chi -2 de Pearson. Le seuil de significativité était de 5\%.

\section{Resultats}

Prévalence de l'obésité : deux cent soixante douze (272) gestantes ont été incluses dans notre étude. La prévalence du surpoids était de 34,9\% et celle de l'obésité de $15,8 \%$ chez les gestantes. (Tableau I)

Age : L'âge moyen des gestantes était de 26,1 $\pm 5,8$ ans avec des extrêmes de 14 et 51 ans. La tranche d'âge comprise entre 25 et 29 ans était la plus représentée avec une fréquence de $33,2 \%$. Les autres tranches d'âge étaient : $<14$ ans : $0,14 \% ; 15$ à 19 ans : $12,6 \% ; 20$ à 24 ans : $25 \% ; 30$ à 34 ans : $19,9 \% ; \geq 35$ ans : $8,9 \%$.

\section{Gestantes obèses et gestantes de poids normal}

\section{* Pathologies survenues au cours de la grossesse}

Le diabète gestationnel était survenu chez $14 \%$ des gestantes obèses contre 3,9\% retrouvé chez les gestantes de poids normal $(\mathrm{p}=0,010)$. Il en est de même pour la prééclampsie( $7 \%$ ) et l'hypertension artérielle gestationnelle $(4,7 \%)$ chez les patientes obèses contre $1,6 \%$ chacun chez les 
patientes de poids normal mais sans lien statistiquement significatif (Tableau II)

\section{* Caractéristiques de l'accouchement}

Le dépassement de terme est retrouvé chez 9,3\% des gestantes obèses. Elles ont accouché des fœus macrosomes dans 9,3\% des cas ( $\mathrm{p}<0,05)$. Le risque d'accouchement prématuré $(18,6 \%)$ et d'accouchement par césarienne $(23,3 \%)$ était plus fréquent chez les gestantes obèses mais sans différence statistiquement significative. Dans la population des obèses ; les mort-nés $(2,3 \%)$, les nouveau-nés réanimés $(11,6 \%)$ et les cas de retard de croissance intra utérin (RCIU), 14\% étaient plus élevés chez que chez les gestantes de poids normal respectivement $0,8 \% ; 8,6 \% ; 9,4 \%$ mais sans différence significative. (Tableau III).

\section{Gestantes en surpoids et gestantes de poids normal}

* Pathologies survenues au cours de la grossesse

Le diabète gestationnel est plus élevé chez les gestantes en surpoids $(17,9 \%)$ que chez les gestantes de poids normal $(3,9 \%)$ de façon significative $(\mathrm{p}=0,00)$. Il en est ainsi pour L' HTA gestationnelle $(2,1 \%)$ et la pré éclampsie $(3,2 \%)$ sont plus fréquentes chez les gestantes en surpoids que celles de poids normal mais sans lien statistique significatif. (Tableau IV)

\section{* Caractéristiques de l'accouchement}

La survenue du dépassement de terme $(3,2 \%)$ et le risque de réanimation du nouveau né $(16,8 \%)$ sont significativement associés aux gestantes en surpoids. Certaines indications de césarienne telles que l'altération du rythme cardiaque fotal $(2,1 \%)$, utérus cicatriciel $(2,1 \%)$ et la suspicion de macrosomie $(2,1 \%)$ sont plus fréquentes chez les gestantes en surpoids que les gestantes de poids normal respectivement 1,6\% pour les deux premières indications et $0 \%$ pour la suspicion de macrosomie mais sans lien significatif. Les gestantes en surpoids ont tendance à accouché d'enfant mort-nés $(1,1 \%)$ et macrosomes $(2,1 \%)$ contre respectivement $0,8 \%$ chacun mais sans différence significative (Tableau V)

\section{Discussion}

La prévalence de l'obésité et du surpoids : dans notre étude, elle était respectivement de $15,8 \%$ et de $34,9 \%$. Au Bénin, l'étude réalisée à Cotonou avait retrouvé une prévalence de l'obésité et du surpoids respectivement $18 \%$ et $30,6 \%$ chez les gestantes (Djrolo et al., 2002). Ces résultats traduisent l'homogénéité de la population, les deux études s'étant déroulées dans le même pays. En France la prévalence de l'obésité chez les gestantes varie entre 7,5\% (Ducarme et al., 2007) et 12\%(Hamon et al.,2005). Ces résultats restent inférieurs au nôtre. Aux Royaumes Unis, une étude avait rapporté une prévalence de l'obésité semblable à la nôtre estimée 
à $15 \%$ ( Zhang $\mathrm{J}$ et al., 2007). D’autres études réalisées aux Etats-Unis et en Irlande ont retrouvé des prévalences de l'obésité supérieures au nôtre respectivement $20 \%$ et 25,2\%( Chu et al., 2009 ; O’Dwyeret al., 2013). Les habitudes alimentaires et le mode de vie très différents dans ces pays peuvent expliquer cette disparité.

\section{Pathologies de la grossesse et caractéristiques de l'accouchement}

Le diabète gestationnel est plus fréquent chez les gestantes obèses et en excès de poids de façon statistiquement significative. Les dysgravidies telles que 1'hypertension artérielle gestationnelle, la prééclampsie sont nettement plus fréquentes chez les femmes obèses et les femmes en surpoids que chez les gestantes de poids normal mais sans lien statistiquement significatif. Ce lien entre les dysgravidies (le diabète gestationnel, 1' HTA gestationnelle, la prééclampsie) et l'excès pondéral a été largement démontré à travers plusieurs études. L'étude réalisée à Cotonou au Bénin avait retrouvé que les femmes en surpoids et obèses avaient tendance à faire plus fréquemment l'hypertension artérielle et la protéinurie sur grossesse avec une différence statistiquement significative. Par contre, le diabète n'avait pas été retrouvé que chez les obèses (Djrolo et al., 2002). D'autres études ont montré qu'il existe une relation linéaire entre l'augmentation de l'IMC et le risque d'apparition d'un diabète gestationnel. En cas de surpoids, le risque relatif est de 1,7 alors qu'il est de 3,6 en cas d'obésité en comparaison à des patientes de poids normal (Deruelle et al., 2012).

Le dépassement de terme a été observé de façon significative chez les gestantes en surcharge pondérale. Par contre, nous n'avions pas retrouvé de risque d'accouchement prématuré associé à l'excès de poids. Djrolo et al. (2002) étaient arrivés à la même conclusion. Par contre, Ducarme et al. (2007) en France ont retrouvé que la prévalence d'accouchement prématuré augmentait avec l'IMC. Concernant le risque de dépassement de terme, d'autres études ont montré qu'il était plus élevé chez les patientes obèses (Weiss et al., 2004 ; Sukalich et al.,2006).

\section{Type d'accouchement}

Nos résultats démontrent que les gestantes obèses et en surpoids sont plus à risque d'accoucher par césarienne mais sans lien statistiquement significatif. Plusieurs travaux retrouvent que les gestantes obèses et en surpoids sont plus à risque d'accoucher par césarienne (Durelle et al., 2011 ; Kumari , 2001 ; Yogev et al., 2008 ; Dempsey et al., 2005). Dempsey et al. (2005) aux Etats-Unis ont montré qu'un IMC supérieur à 30 multipliait par trois à six le risque de césarienne. Le risque de césarienne chez ces femmes serait en rapport avec le risque plus élevé de prééclampsie, de macrosomie fœtale et de dystocie dynamique. Dans notre étude l'indication de césarienne 
prédominante chez les gestantes obèses était 1'utérus cicatriciel ; ce qui sous-entend qu'elles avaient antérieurement bénéficié d'une césarienne dont les indications n'ont pu être précisées. Chez les gestantes en surpoids, la dystocie dynamique était la plus fréquente indication de césarienne. Ducarme et al en France retrouvaient que la prévalence de dystocie dynamique augmentait chez les gestantes obèses et en surpoids comparativement aux gestantes de poids normal. Ce qui faisait que les gestantes en excès pondéral qui accouchaient par voie basse bénéficiaient plus fréquemment d'une direction du travail d'accouchement (Ducarme et al., 2007).

\section{Caractéristiques néonatales en fonction de l'IMC}

$\mathrm{Au}$ décours de notre étude, nous avons retrouvé que les gestantes en surcharge pondérale étaient plus à risque de donner naissance à des mort-nés mais sans lien statistiquement significatif. Concernant les nouveau-nés réanimés à la naissance ; la prévalence de nouveau-nés ayant un APGAR $\leq 7$ à la première minute et donc ayant bénéficié d'une réanimation à la naissance était plus élevée chez les gestantes en surpoids et chez les obèses comparativement aux gestantes de poids normal. L'étude au Bénin avait abouti à la même conclusion (Djrolo et al., 2002). Pour Cedergren et al. (2004), en cas d'obésité ou de surpoids, le risque de détresse néonatale est multiplié par 2,52, d'inhalation méconiale par 2,85 et de décès néonatal par 3,41 . Toutes ces données sont importantes afin de mieux prendre en charge les nouveau-nés issus de ces mères, ce d'autant plus que leurs accouchements sont déclenchés après maturation cervicale pour macrosomie fœtale associée ou non à un diabète (N'Diaye et al., 2005). Aussi, une étude réalisée en Suède, à partir du registre de naissance national suédois a-t-elle noté que le risque de mort fœtal in utero (MFIU) était doublé chez les patientes présentant un IMC supérieur ou égal à $30 \mathrm{~kg} / \mathrm{m}^{2}$ (Deruelle , 2011).

Concernant le poids du nouveau-né, nos résultats montrent que la fréquence de macrosomies augmente avec l'IMC. Plusieurs études ont démontré que les femmes en excès de poids surtout les obèses étaient plus fréquemment à risque d'accoucher des nouveau-nés macrosomes (Ducarme et al., 2007 ; Djrolo et al., 2002 ; Hamon et al., 2005 ; Kumari , 2001). La fréquence de fœtus macrosomes chez les patientes obèses est plus élevée (15-30 \%) avec un risque relatif variant entre 2 et 3,82 et un risque de dystocie des épaules multiplié par 3,14 (Ducarme et al., 2007). Dans notre étude, concernant le RCIU, la prévalence des gestantes en surpoids ayant donné naissance à des nouveau- nés ayant un retard de croissance intra utérin $(7,4 \%)$ était moins élevée que chez les gestantes de poids normal $(9,4 \%)$ mais sans lien statistiquement significatif. Djrolo et al. (2002) au Bénin, N'Diaye et al., (2005) au Sénégal et Ducarme et al. (2007) en France ont tous conclu au décours de chacune de leur étude que la fréquence de 
nouveau-nés ayant un RCIU était moindre chez les femmes obèses que chez les femmes de poids normal. Certaines études plus récentes ont même exclut les facteurs confondants possibles que sont: la gémellité, les anomalies de cordon et du placenta, les maladies maternelles (drépanocytose, malnutrition, cardiopathie, intoxication tabagique), les embryofoetopathies infectieuses, les anomalies constitutionnelles, (Merger et al., 2001) et ont confirmé que l'excès de poids n'augmentait pas le risque de RCIU. Certains ont même affirmé que l'excès de poids chez la femme protège contre la mise au monde de nouveau-nés hypotrophiques (Weiss et al., 2004 ; Kumari , 2001). Nous pouvons expliquer cette différence de résultat dans notre étude par le faite que nous n'avons pas écarté les facteurs confondants.

\section{Conclusion}

L'excès de poids est la résultante d'une mauvaise hygiène alimentaire et de la sédentarité. Au cours de la grossesse, elle est associée à un risque élevé de complications obstétricales. Notre étude semble confirmer que l'obésité et le surpoids sont responsables de complications obstétricales devant faire considérer ces grossesses comme des grossesses à risque.

\section{References:}

Cedergren MI. 2004. Maternalmorbidobesity and the risk of adverse pregnancyoutcome. ObstetGynecol ;103:219-24.

Cohen E. 2010. L'image du corps chez les sénégalais : application à l'étude de l'obésité dans le contexte de la transition des modes de vie. L'anthropologie des vivants : objets et méthodes ; 30: 52-57.

Chu SY, Kim SY, Bish CL. 2009. Prepregnancyobesityprevalence in the United States, 2004-2005. Matern Child Health J ; 13 : 614-20.

Dempsey JC, Ashiny Z, Qiu CF, Miller RS, Sorensen TK, Williams MA. 2005. Maternalpre-pregnancyoverweightstatus and obesity as riskfactors for cesareandelivery. J MaternFetalNeonatal Med ;17:179-85.

Deruelle P. 2011. Obesity and pregnancy. GynecolObstetFertil ; 39 : 100-5. Deruelle P, Vambergue A. 2012. Diabète et obésité : un défi obstétrical !

Médecine des maladies Métaboliques ; $6: 294-299$.

Djrolo F, Megnigbeto Obey A, de Souza J, Takpara I, Santos P, Alihonou E. 2002. Influence du poids maternel sur l'évolution de la grossesse à Cotonou (Bénin). J GynecolObstetBiolReprod ; 31 : 243-247.

Ducarme G, Rodrigues A, Aissaoui F, Davitian C, Pharisien I, Uzan M. 2007. Grossesse des patientes obèses : quels risques faut-il craindre? Gynécologie Obstétrique \& Fertilité ; 35 :19-24.

Eicholzer M. 2006. Is low or high body weight associated with an increased risk of neural tube defects? SchweizRundsch Med Prax ; 95: 2019-26. 
Grossetti E, Beucher G, Régeasse A, Lamendour N, Herlicoviez M, Dreyfus M. 2004. Complications obstétricales de l'obésité morbide. J GynecolObstetBiol

Reprod ; 33 : 739-744.

Hamon C, Fanello S, Catala L, Parot E. 2005. Conséquences de l'obésité maternelle sur le déroulement du travail et de l'accouchement. J GynecolObstetBiolReprod ; 34 : 109-114.

Kumari AS. 2001. Pregnancyoutcome in womenwithmorbidobesity. Int J GynaecolObstet ; $73: 101-7$.

Merger R, Levy J, Melchior J. 2001. Précis d'obstétrique.6ème édition. Paris. Masson.

Ndiaye O, Sylla A, Cissé CT, Guèye M, Ndabashinzé P, Ouattara A. 2005. Influence de l'excès de poids maternel sur le poids de naissance d'une population de nouveau-nés à terme au Sénégal. Journal de pédiatrie et de puériculture ; $18: 33-37$.

O'Dwyer V, O'Toole F, Darcy S, Farah N, Kennelly MM, Turner MJ. 2013. Maternal obesity and gestational weight gain. Journal of Obstetrics and Gynaecology ; 33: 671-674.

Puech F. 2010. Recommandations pour la pratique clinique. le diabète gestationnel .J GynecolObstetBiolReprod ; 39 : 1-342.

Sukalich S, Mingione JM, Glantz JC. 2006. Obstetricoutcomes in overweight and obese adolescents. American Journal of Obstetrics and Gynaecology ; $195: 851-5$.

Weiss JL, Malone FD, Emig D. 2004. Obesity, obstetric complications and cesareandelivery rate- a population-based screening study. Am J ObstetGynecol ; $190: 1091$ -

World Health Organization. 1997. Preventing and Managing the global Epidemic. Report on WHO consultation on Obesity, Geneva, 3-5 June 1997. WHO/NUT/NDC/98.I.Geneva: WHO.

Yogev Y, Langer O. 2008. Pregnancyoutcome in obese and morbidly obese gestationaldiabeticwomen. European Journal of Obstetrics\&Gynecology and Reproductive Biology ; 137 : 21-26.

Zhang J, Bricker L, Wray S. 2007. Poor uterinecontractility in obese women. BJOG ; $114: 343-8$.

\section{Liste des tableaux}

Tableau I: Répartition des femmes enquêtées en fonction de l'IMC (Parakou, 2015)

\begin{tabular}{|c|c|c|}
\hline Tranche d'IMC ( $\left.\mathrm{kg} / \mathrm{m}^{2}\right)$ & Effectifs & Pourcentage(\%) \\
\hline$<18,5$ & 6 & 2,2 \\
\hline $18,5-24,9$ & 128 & 47,1 \\
\hline $25-29,9$ & 95 & 34,9 \\
\hline$\geq 30$ & 43 & 15,8 \\
\hline Total & 272 & 100,0 \\
\hline
\end{tabular}


Tableau II: Répartition des pathologies survenues au cours de la grossesse des gestantes obèses et des gestantes de poids normal (Parakou 2015)

\begin{tabular}{cccccc}
\hline \multirow{2}{*}{ Pathologies } & \multicolumn{3}{c}{$\begin{array}{c}\text { Obésité } \\
(\mathrm{n}=43)\end{array}$} & \multicolumn{3}{c}{$\begin{array}{c}\text { Normal } \\
(\mathrm{n}=128)\end{array}$} & $\mathrm{p}$ \\
\cline { 2 - 5 } & $\mathrm{n}$ & $\%$ & $\mathrm{n}$ & $\%$ & \\
\hline Eclampsie & 0 & 0,0 & 2 & 1,6 & 0,415 \\
Diabète gestationnel & 6 & 14,0 & 5 & 3,9 & 0,010 \\
HTA gestationnel & 3 & 7,0 & 2 & 1,6 & 0,060 \\
MFIU & 0 & 0,0 & 1 & 0,8 & 0,565 \\
Oligoamnios & 0 & 0,0 & 4 & 3,1 & 0,246 \\
Prééclampsie & 2 & 4,7 & 2 & 1,6 & 0,235 \\
\hline
\end{tabular}

Tableau III : Caractéristiques de l'accouchement des gestantes obèses et gestantes de poids normal (Parakou, 2015)

\begin{tabular}{|c|c|c|c|c|c|}
\hline & \multicolumn{2}{|c|}{ Obésité $(n=43)$} & \multicolumn{2}{|c|}{ Normal $(n=128)$} & \multirow{2}{*}{$\mathrm{p}$} \\
\hline & $\mathrm{n}$ & $\%$ & $\mathrm{n}$ & $\%$ & \\
\hline \multicolumn{6}{|l|}{$\begin{array}{c}\text { Terme à } \\
\text { l'accouchement }\end{array}$} \\
\hline Normal & 31 & 72,1 & 114 & 89,1 & 0,007 \\
\hline $\begin{array}{l}\text { Dépassement de } \\
\text { terme }\end{array}$ & 4 & 9,3 & 0 & 0 & 0 \\
\hline $\begin{array}{c}\text { Prématurité } \\
\text { Voie } \\
\text { d'accouchement }\end{array}$ & 8 & 18,6 & 14 & 10,9 & 0,09 \\
\hline $\begin{array}{l}\text { Voie basse } \\
\text { naturelle }\end{array}$ & 11 & 25,5 & 30 & 23,4 & 0,776 \\
\hline Césarienne & 10 & 23,3 & 17 & 13,3 & 0,106 \\
\hline $\begin{array}{l}\text { Voie basse dirigée } \\
\text { Indication de } \\
\text { césarienne }\end{array}$ & 22 & 51,2 & 81 & 63,3 & 0,216 \\
\hline Prééclampsie & 0 & 0 & 0 & 0 & - \\
\hline Altération du RCF & 2 & 4,7 & 2 & 1,6 & 0,716 \\
\hline $\begin{array}{l}\text { Dystocie } \\
\text { dynamique }\end{array}$ & 3 & 7 & 7 & 5,5 & 0,31 \\
\hline Eclampsie & 0 & 0 & 2 & 1,6 & 0,415 \\
\hline Utérus cicatriciel & 6 & 14 & 2 & 1,6 & 0,139 \\
\hline $\begin{array}{l}\text { Suspicion de } \\
\text { macrosomie }\end{array}$ & 4 & 9,3 & 0 & 0 & 0,267 \\
\hline Oligoamnios & 0 & 0 & 4 & 3,1 & 0,246 \\
\hline $\begin{array}{l}\text { Caractéristiques } \\
\text { du nouveau-né }\end{array}$ & & & & & \\
\hline Vivant & 27 & 62,8 & 103 & 80,5 & 0,02 \\
\hline Mort-né & 1 & 2,3 & 1 & 0,8 & 0,404 \\
\hline Réanimation & 5 & 11,6 & 11 & 8,6 & 0,453 \\
\hline Macrosomie & 4 & 9,3 & 1 & 0,8 & 0,003 \\
\hline RCIU & 6 & 14 & 12 & 9,4 & 0,37 \\
\hline
\end{tabular}


Tableau IV: Répartition des pathologies survenues au cours de la grossessedes gestantes en surpoids et les gestantes de poids normal (Parakou 2015)

\begin{tabular}{cccccc}
\hline \multirow{2}{*}{ Pathologies } & \multicolumn{2}{c}{ Surpoids $(\mathrm{n}=95)$} & Normal $(\mathrm{n}=128)$ & \multirow{2}{*}{$\mathrm{p}$} \\
\cline { 2 - 5 } & $\mathrm{n}$ & $\%$ & $\mathrm{n}$ & $\%$ & \\
\hline Eclampsie & 1 & 1,1 & 2 & 1,6 & 0,758 \\
Diabète gestationnel & 17 & 17,9 & 5 & 3,9 & 0,000 \\
HTAG & 2 & 2,1 & 2 & 1,6 & 0,744 \\
MFIU & 0 & 0,0 & 1 & 0,8 & 0,393 \\
Oligoamnios & 0 & 0,0 & 4 & 3,1 & 0,085 \\
Prééclampsie & 3 & 3,2 & 2 & 1,6 & 0,410 \\
\hline
\end{tabular}

Tableau V : Gestantes en surpoids et gestantes de poids normal (Parakou, 2015)

\begin{tabular}{|c|c|c|c|c|c|}
\hline & \multicolumn{2}{|c|}{ Surpoids $(\mathrm{n}=95)$} & \multicolumn{2}{|c|}{ Normal $(n=128)$} & \multirow[b]{2}{*}{$\mathrm{p}$} \\
\hline & $\mathrm{n}$ & $\%$ & $\mathrm{n}$ & $\%$ & \\
\hline \multicolumn{6}{|l|}{ Terme à } \\
\hline Normal & 82 & 86,3 & 114 & 98,1 & 0,534 \\
\hline $\begin{array}{l}\text { Dépassement de } \\
\text { terme }\end{array}$ & 3 & 3,2 & 0 & 0 & 0,04 \\
\hline $\begin{array}{c}\text { Prématuré } \\
\text { Voie } \\
\text { d'accouchement }\end{array}$ & 10 & 10,5 & 14 & 10,9 & 0,965 \\
\hline $\begin{array}{l}\text { Voie basse } \\
\text { naturelle }\end{array}$ & 30 & 31,6 & 30 & 23,4 & 0,175 \\
\hline Césarienne & 13 & 13,7 & 17 & 13,3 & 0,877 \\
\hline $\begin{array}{l}\text { Voie basse } \\
\text { dirigée }\end{array}$ & 52 & 54,7 & 81 & 63,3 & 0,55 \\
\hline $\begin{array}{l}\text { Indication de } \\
\text { césarienne }\end{array}$ & & & & & \\
\hline Prééclampsie & 0 & 0 & 0 & 0 & - \\
\hline $\begin{array}{l}\text { Altération du } \\
\text { RCF }\end{array}$ & 2 & 2,1 & 2 & 1,6 & 0,341 \\
\hline $\begin{array}{l}\text { Dystocie } \\
\text { dynamique }\end{array}$ & 5 & 5,3 & 7 & 5,5 & 0,496 \\
\hline Eclampsie & 1 & 1,1 & 2 & 1,6 & 0,872 \\
\hline Utérus cicatriciel & 2 & 2,1 & 2 & 1,6 & 0,341 \\
\hline $\begin{array}{l}\text { Suspicion de } \\
\text { macrosomie }\end{array}$ & 2 & 2,1 & 0 & 0 & 0,759 \\
\hline Oligoamnios & 0 & 0 & 4 & 3,1 & 0,128 \\
\hline $\begin{array}{l}\text { Caractéristiques } \\
\text { du nouveau - né }\end{array}$ & & & & & \\
\hline Vivant & 69 & 72,6 & 103 & 80,5 & 0,168 \\
\hline Mort-né & 1 & 1,1 & 1 & 0,8 & 0,818 \\
\hline $\begin{array}{c}\text { Réanimation à la } \\
\text { naissance }\end{array}$ & 16 & 16,8 & 11 & 8,6 & 0,05 \\
\hline Macrosomie & 2 & 2,1 & 1 & 0,8 & 0,384 \\
\hline RCIU & 7 & 7,4 & 12 & 9,4 & 0,631 \\
\hline
\end{tabular}

